\title{
TU/e EmonOWEN

\section{Effects of antenna correlation and mutual coupling on the carrier frequency offset estimation in MIMO systems}

\section{Citation for published version (APA):}

Wu, Y., Bergmans, J. W. M., \& Attallah, S. (2010). Effects of antenna correlation and mutual coupling on the carrier frequency offset estimation in MIMO systems. In International Conference on Wireless Communication, Networking and Mobile Computing (Wicom), Chengdu, 23-25 Sept. 2010 (pp. 1-4). Institute of Electrical and Electronics Engineers. https://doi.org/10.1109/WICOM.2010.5600570

DOI:

10.1109/WICOM.2010.5600570

Document status and date:

Published: 01/01/2010

\section{Document Version:}

Publisher's PDF, also known as Version of Record (includes final page, issue and volume numbers)

\section{Please check the document version of this publication:}

- A submitted manuscript is the version of the article upon submission and before peer-review. There can be important differences between the submitted version and the official published version of record. People interested in the research are advised to contact the author for the final version of the publication, or visit the $\mathrm{DOI}$ to the publisher's website.

- The final author version and the galley proof are versions of the publication after peer review.

- The final published version features the final layout of the paper including the volume, issue and page numbers.

Link to publication

\section{General rights}

Copyright and moral rights for the publications made accessible in the public portal are retained by the authors and/or other copyright owners and it is a condition of accessing publications that users recognise and abide by the legal requirements associated with these rights.

- Users may download and print one copy of any publication from the public portal for the purpose of private study or research.

- You may not further distribute the material or use it for any profit-making activity or commercial gain

- You may freely distribute the URL identifying the publication in the public portal.

If the publication is distributed under the terms of Article 25fa of the Dutch Copyright Act, indicated by the "Taverne" license above, please follow below link for the End User Agreement:

www.tue.nl/taverne

Take down policy

If you believe that this document breaches copyright please contact us at:

openaccess@tue.nl

providing details and we will investigate your claim. 


\title{
Effects of Antenna Correlation and Mutual Coupling on the Carrier Frequency Offset Estimation in MIMO Systems
}

\author{
Yan Wu*, J.W.M. Bergmans*, and S. Attallah ${ }^{\dagger}$ \\ ${ }^{*}$ Department of Electrical Engineering \\ Eindhoven University of Technology (TU/e), The Netherlands \\ $\dagger$ School of Science and Technology, SIM University, Singapore
}

\begin{abstract}
In practical multiple-input multiple-output (MIMO) systems, due to the close proximity among the antennas, the channels between different transmit and receive antennas are spatially correlated. Besides spatial correlation, closely placed antennas also experience mutual coupling among the antennas, which is due to the interaction of electro-magnetic fields at different antennas. In this paper, we present a study on the effects of spatial correlation and mutual coupling and in particular, their impacts on the performance of the carrier frequency offset (CFO) estimation in MIMO systems. The simulation results show that spatial correlation degrades the performance of the CFO estimation. Mutual coupling has two effects. Firstly it reduces the spatial correlation, which is beneficial. On the other hand, it also reduces the power of the desired signal, which is detrimental. Simulations results show that the combined effects of mutual coupling introduces additional degradation on the CFO estimation.
\end{abstract}

\section{INTRODUCTION}

Multiple-input multiple-output (MIMO) systems increase the information capacity of rich scattering wireless fading channels enormously by using multiple antennas at both the transmitter and the receiver [1]. In the study of MIMO systems, a common assumption used in many works is that the channel responses between different transmit and receive antennas are statistically independent. In practice, due to the close proximity among the antennas, the channel responses are spatially correlated. The spatial correlation is related to the propagation environment of the wireless signals. Besides this spatial correlation, the electromagnetic (EM) fields at closelyplaced antennas also interact with each other and cause mutual coupling among the antennas [2] [3]. The effect of antenna correlation and mutual coupling on the capacity of the MIMO channel was studied in [4] [5]. It was shown that both spatial correlation and mutual coupling reduces the capacity of MIMO channels.

In this paper, we present a study of the effects of antenna correlation and mutual coupling on the carrier frequency offset (CFO) estimation in MIMO systems. We first present a mathematical model of the spatial correlation and show that it is dependent on the distributions of angle of arrival (AOA) at the receive antennas and the angle of departure (AOD) at the transmit antennas. These distributions can be characterized by a distribution function called power angular spectrum (PAS). We study two specific PAS distributions, namely the uniform
PAS, which is the most commonly used, and the Laplacian PAS, which more accurately describes the indoor propagation environments [6] [7]. It is found that Laplacian PAS introduces more spatial correlation due to its narrower angular spread. Computer simulations show that higher spatial correlation leads to larger degradation in the CFO estimation compared to independent and identically-distributed (i.i.d.) MIMO channels. We also model the effect of mutual coupling. We see that mutual coupling firstly reduces the spatial correlation, which is beneficial. On the other hand, mutual coupling also reduces the power of the desired signal, which is detrimental. Simulations results show that the combined effects of mutual coupling introduces additional degradation on the CFO estimation.

\section{EFFECTS OF SPATIAL CORRELATION}

In this section, we study the spatial correlation among antennas related to the propagation environment and also its effects on the performance of the CFO estimation in MIMO systems. As the correlation effect is similar for different paths in a multi-path channel, in this section, we only consider the effect on one path, i.e. we only consider a flat fading channel. For a MIMO system with $N_{t}$ transmit and $N_{r}$ receive antennas, the received signal in flat fading channels can be written as

$$
\mathbf{r}=\mathbf{H s}+\mathbf{n}
$$

where $\mathbf{s}$ is the transmitted signal from $N_{t}$ transmit antennas, $\mathbf{H}$ is the $N_{r} \times N_{t}$ channel matrix with the $i, j$ th element $H_{i, j}$ as the channel response between the $j$ th transmit antenna and the $i$ th receive antenna and $\mathbf{n}$ is the AWGN noise. In practice, MIMO channels are correlated in the spatial domain. Such correlation can be modeled as [8]

$$
\mathbf{H}=\left[\mathbf{R}_{\mathrm{rx}}\right]^{1 / 2} \mathbf{H}_{\mathrm{iid}}\left(\left[\mathbf{R}_{\mathrm{tx}}\right]^{1 / 2}\right)^{T},
$$

where $\mathbf{H}_{\mathrm{iid}}$ is the channel matrix generated using i.i.d. zeromean complex Gaussian random variables. The transmit and receive correlation matrices are denoted as $\mathbf{R}_{\mathrm{tx}}$ and $\mathbf{R}_{\mathrm{rx}}$ respectively.

To simplify the analysis, we only look at the spatial correlation at the receive antennas, while assuming zero correlation among the transmit antennas. The spatial correlation is related to the propagation environment and is dependent 


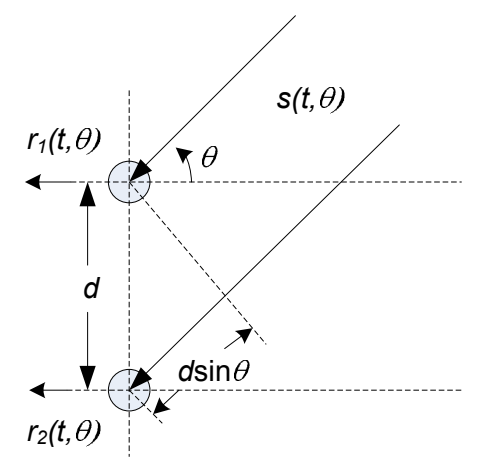

Fig. 1. Received signal for a two-element antenna array spaced $d$ for a plane wave impinging at angle $\theta$.

on the distributions of angle of arrival (AOA) and angle of departure (AOD), which are specified by the power angular spectrum (PAS). For ease of illustration, we consider a twoantenna receiver. The results can be extended to more than two antennas straight-forwardly. Figure 1 illustrates a receiver with two antennas spaced $d$ meters apart. There is a plane wave $(s(t, \theta))$ impinging at an AOA of $\theta$. The received signal at the $i$ th receive antenna can be expressed as [9]

$$
r_{i}(t, \theta)=\sqrt{G_{i}(\theta)} s(t, \theta) e^{j 2 \pi\left[f_{c}+(i-1) \frac{d}{\lambda} \sin \theta\right]},
$$

where $s(t, \theta)$ is the impinging signal, $t$ is time and $\theta$ is the AOA of $s(t, \theta)$. The carrier frequency is denoted as $f_{c}, d$ is the spacing between the antennas and $\lambda$ is the wavelength. The power gain of the $i$ th antenna at angle $\theta$ is denoted as $G_{i}(\theta)$. The covariance matrix of the received signal can be written as

$$
\mathrm{R}_{r}=\left[\begin{array}{cc}
P_{1} & \mathrm{E}_{t, \theta}\left(r_{1}(t, \theta) r_{2}^{*}(t, \theta)\right) \\
\mathrm{E}_{t, \theta}\left(r_{1}^{*}(t, \theta) r_{2}(t, \theta)\right) & P_{2}
\end{array}\right],
$$

where we use $\mathrm{E}_{t, \theta}$ to denote statistical expectation taken over both time $t$ and the angle $\theta$. The received signal power at each receive antenna is given by $P_{i}=\mathrm{E}_{t, \theta}\left(G_{i}(\theta)|s(t, \theta)|^{2}\right.$. The correlation coefficients of the received signals at the two antennas are defined as

$$
\rho_{1,2}=\frac{\mathrm{E}_{t, \theta}\left[r_{1}(t, \theta) r_{2}^{*}(t, \theta)\right]-\mathrm{E}_{t, \theta}\left[r_{1}(t, \theta)\right] \mathrm{E}_{t, \theta}\left[r_{2}^{*}(t, \theta)\right]}{\sqrt{P_{1} P_{2}}} .
$$

We assume that the impinging signal $s(t, \theta)$ has zero mean over all angles, so that $\mathrm{E}_{t, \theta}\left[r_{1}(t, \theta)\right]=0$. Similarly we have $\mathrm{E}_{t, \theta}\left[r_{2}(t, \theta)\right]=0$. Therefore, the correlation coefficient can be simplified to

$$
\rho_{1,2}=\frac{\mathrm{E}_{t, \theta}\left[r_{1}(t, \theta) r_{2}^{*}(t, \theta)\right]}{\sqrt{P_{1} P_{2}}},
$$

and the covariance matrix can be re-written as

$$
\mathbf{R}_{r}=\sqrt{P_{1} P_{2}}\left[\begin{array}{cc}
1 & \rho_{1,2} \\
\rho_{1,2}^{*} & 1
\end{array}\right] .
$$

Denoting $D=2 \pi \frac{d}{\lambda}$, we can write

$$
\begin{aligned}
& \mathrm{E}_{t, \theta}\left[r_{1}(t, \theta) r_{2}^{*}(t, \theta)\right] \\
= & \mathrm{E}_{\theta}\left[\sqrt{G_{1}(\theta) G_{2}(\theta)} \mathrm{E}_{t}\left(|s(t, \theta)|^{2}\right) e^{-j D \sin \theta}\right] .
\end{aligned}
$$

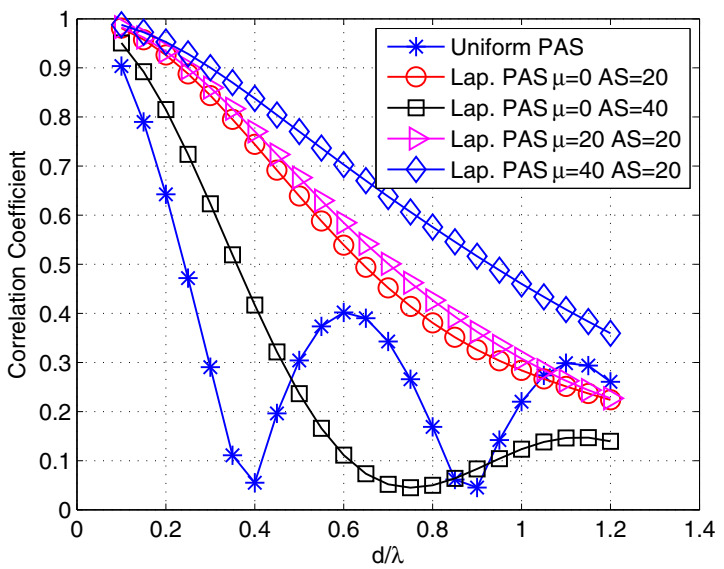

Fig. 2. Correlation coefficients for different angular spreads and different mean AOA values.

It is reasonable to assume that different antenna elements in an antenna array have the same radiation pattern, i.e. $G_{1}(\theta)=$ $G_{2}(\theta)=G(\theta)$. In this case, we also have $P_{1}=P_{2}=P$. We use $P_{s}(\theta)=\mathrm{E}_{t}\left(|s(t, \theta)|^{2}\right)$ to denote the power of the impinging signal from angle $\theta$. We also assume that the power of the impinging signal is independent of $\theta$ and hence we will use $P_{s}$ instead. The PAS of the received signal at angle $\theta$ is denoted as $\operatorname{PAS}(\theta)$. With these, we can rewrite the correlation coefficient in (6) as

$$
\rho_{1,2}=\frac{\int_{-\pi}^{\pi} G(\theta) \operatorname{PAS}(\theta) e^{-j D \sin \theta} d \theta}{\int_{-\pi}^{\pi} G(\theta) \operatorname{PAS}(\theta) d \theta} .
$$

A common assumption used in the analysis of the spatial correlation is that the AOA is uniformly distributed between 0 and 360 degrees, i.e. $\operatorname{PAS}(\theta)=1 /(2 \pi)$ for all $\theta$ values. In this case, the correlation coefficient is given by

$$
\rho_{1,2}=\frac{\int_{-\pi}^{\pi} e^{-j D \sin \theta} G(\theta) d \theta}{\int_{-\pi}^{\pi} G(\theta) d \theta} .
$$

Moreover, if the antennas are omni-directional, i.e. $G(\theta)=G$ for all the angles, then $\rho_{1,2}=J_{0}(D)$ where $J_{0}$ is the Bessel function of the first kind and order 0 .

It was found in [6] [7] that the PAS for indoor environments closely matches a Laplacian distribution given by

$$
\operatorname{PAS}(\theta)=\frac{1}{\sqrt{2} \sigma} \exp \left(-\left|\frac{\sqrt{2}(\theta-\mu)}{\sigma}\right|\right)
$$

where $\sigma$ is the angular spread, $\mu$ is the mean AOA and both are in degrees. For this PAS, there is no closed-form solution for the correlation coefficients. Therefore, we use numerical integration to calculate $\rho_{1,2}$.

Figure 2 shows a comparison of the correlation coefficients for uniform PAS and Laplacian PAS. For Laplacian PAS, we also compare the correlation coefficients for different mean AOA and angular spread (AS) values for omni-directional antennas. From the figure, we can see that the correlation 


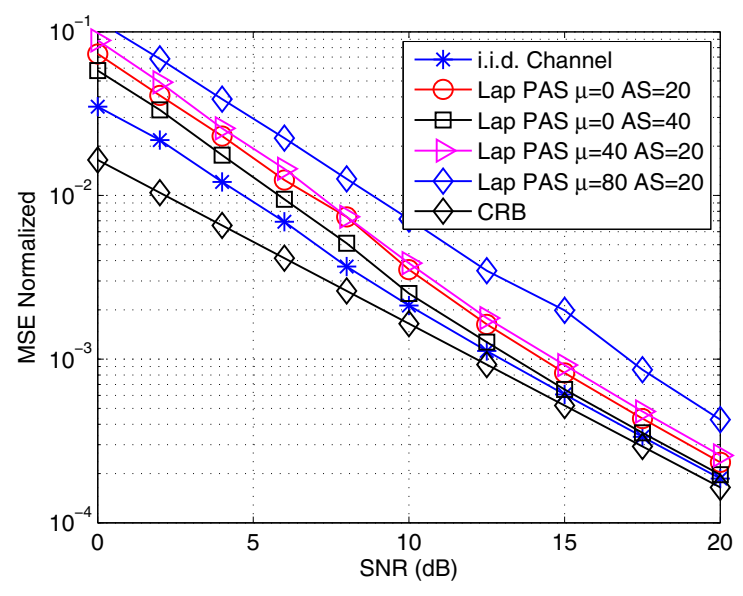

Fig. 3. MSE of CFO estimation for Laplacian PAS with different angular spreads and different mean AOA values.

coefficients for Laplacian PAS are much higher than a uniform PAS. For a fixed mean AOA of $\mu=0^{\circ}$, we can see that larger angular spreads lead to smaller correlations. For the same angular spread of $\mathrm{AS}=20^{\circ}$, the correlation becomes larger when the mean AOA becomes larger.

We use computer simulations to study the performance of CFO estimation for Laplacian PAS with different mean AOA and angular spread values for a $2 \times 2$ MIMO system in flat fading channels. The spacing between the 2 receive antennas is $0.5 \lambda$. For CFO estimation, we use the timedomain maximum likelihood estimator using periodic training sequences proposed in [10]. We use two periods of length16 training sequences. Figure 3 shows the MSE of CFO estimation for different mean AOA and AS values. We also plotted the Cramer-Rao bound (CRB) for the CFO estimation for i.i.d. MIMO channels. From the figure, we can see that the performance of CFO estimation is degraded compared to that of the i.i.d. channel due to the spatial correlation. For a fixed mean AOA values of $\mu=0^{\circ}$, the degradation is larger for smaller angular spread values due to the larger spatial correlation. For a fixed angular spread of $\mathrm{AS}=20^{\circ}$, we can see that the performance degradation is larger for larger mean AOA values. This is because, as shown in Figure 2, for a fixed angular spread, the spatial correlation is larger for larger mean AOA values.

\section{EFFECTS OF MUTUAL COUPLING}

Mutual coupling among different antenna elements in an antenna array is caused by the interactions of the EM waves received at different antenna elements. This effect is related to the antenna array and is independent of the propagation environment. The effects of mutual coupling were studied in [2] and [3]. It was shown that with mutual coupling among different antennas, the effective channel can be re-written as

$$
\mathbf{H}=\left[\mathbf{C}_{\mathrm{rx}} \mathbf{R}_{\mathrm{rx}} \mathbf{C}_{\mathrm{rx}}^{H}\right]^{1 / 2} \mathbf{H}_{\mathrm{iid}}\left(\left[\mathbf{C}_{\mathrm{tx}} \mathbf{R}_{\mathrm{tx}} \mathbf{C}_{\mathrm{tx}}^{H}\right]^{1 / 2}\right)^{T},
$$

where $\mathbf{C}_{\mathrm{rx}}$ and $\mathbf{C}_{\mathrm{tx}}$ are the coupling matrices for the receiver and transmitter respectively. In this section, for simplicity of illustration, we only consider the effects of propagation environments and mutual coupling at the receiver. In this case, the effective channel can be simplified to

$$
\mathbf{H}=\left[\mathbf{C R C}^{H}\right]^{1 / 2} \mathbf{H}_{\mathrm{iid}} .
$$

Here we dropped the subscript of rx to simplify the notation. In [11], it was shown that mutual coupling reduces the spatial correlation between the antennas. On the other hand, mutual coupling also results in power loss on the desired signal when the two antennas are placed too close [5]. Next, we look at the overall effects of mutual coupling on the performance of CFO estimation in MIMO systems.

Let us consider a receiver with two dipole antennas. From [2], the coupling matrix in (13) can be calculated as

$$
\mathbf{C}=\left(Z_{\text {load }}+Z_{s}\right)\left[\begin{array}{cc}
Z_{\text {load }}+Z_{s} & Z_{m} \\
Z_{m} & Z_{\text {load }}+Z_{s}
\end{array}\right]^{-1} .
$$

In this study, we assume that the loading impedance $Z_{\text {load }}$ is matched to the self impedance $Z_{s}$ of the antenna, i.e. $Z_{\text {load }}=Z_{s}^{*}$. The mutual impedance $Z_{m}$, which is due to the mutual coupling, is a function of the dipole length $l$, the antenna spacing $d$ and the antenna placement configuration. The mutual impedance can be calculated numerically using the induced Electromagnetic Fields (EMF) method [12]. Combining the effect of coupling with the spatial correlation related to the propagation environment, we have

$$
\begin{aligned}
\mathbf{C R C}^{\mathbf{H}} & =\left[\begin{array}{ll}
C_{1,1} & C_{1,2} \\
C_{2,1} & C_{2,2}
\end{array}\right]\left[\begin{array}{cc}
1 & \rho_{1,2} \\
\rho_{2,1} & 1
\end{array}\right]\left[\begin{array}{ll}
C_{1,1}^{*} & C_{2,1}^{*} \\
C_{1,2}^{*} & C_{2,2}^{*}
\end{array}\right] \\
& =P\left[\begin{array}{cc}
1 & \tilde{\rho}_{1,2} \\
\tilde{\rho}_{2,1} & 1
\end{array}\right] .
\end{aligned}
$$

From (15), we can see that there are two effects from mutual coupling. Firstly, mutual coupling changes the spatial correlation. Secondly, the received signal power is scaled by $P$.

The effective correlation $\left(\left|\tilde{\rho}_{1,2}\right|\right)$ as a function of the antenna spacing for a 2-antenna receiver is depicted in Figure 4. Here, we assume two dipole antennas with length $l=0.5 \lambda$ placed side by side. For such antennas, the self-impedance is $Z_{s}=(73+j 42) \Omega[12]$. We also assume that the AOA has a uniform distribution from 0 to $360^{\circ}$. We can see that with the effect of mutual coupling, the spatial correlation between the antennas is reduced. The power $P$ as a function of the antenna spacing is shown in Figure 5. The plot shows that the system suffers significant power loss if the two antennas are spaced too closely. The power loss becomes insignificant when the antenna spacing is about $1 \lambda$. In summary, the effect of mutual coupling is two-fold. Firstly it reduces the spatial correlation between the antennas, which is a desirable effect. On the other hand, it introduces extra power loss, which is undesirable.

We used computer simulations to investigate the combined effect of mutual coupling on the performance of the CFO estimation. We simulated a $2 \times 2$ MIMO system for flat fading channels. We assume the transmit antennas are independent 


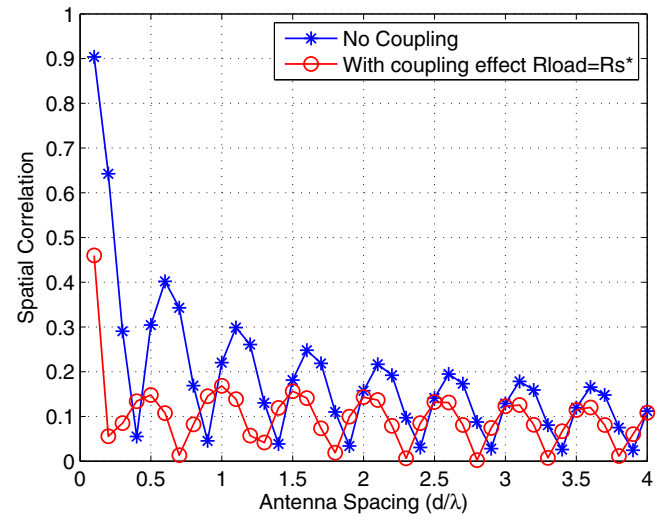

Fig. 4. Effective spatial correlation due to coupling for two $\lambda / 2$ dipole antennas with $Z_{\text {load }}=Z_{s}^{*}$.

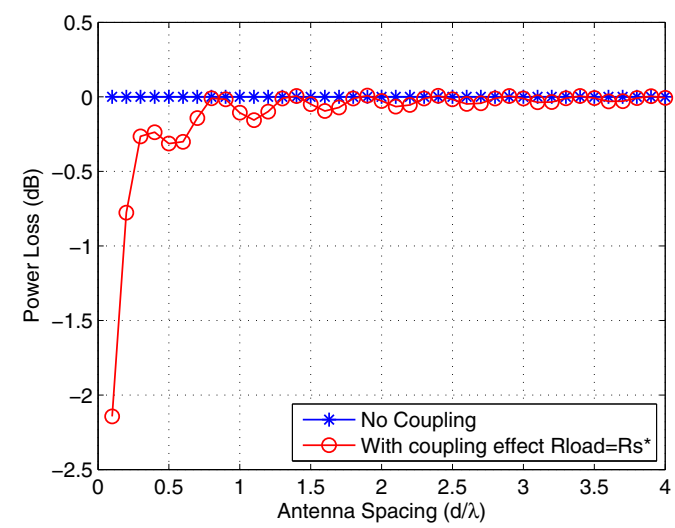

Fig. 5. Power loss due to coupling for two $\lambda / 2$ dipole antennas with $Z_{\text {load }}=$ $Z_{s}^{*}$

and only consider the effect of coupling at the receiver. The spacing between two receive antenna is $0.5 \lambda$. We also assume an uniformly distributed $\mathrm{AOA}$ at the receiver. We use the same training sequence and $\mathrm{CFO}$ estimator as those used in obtaining Figure 3. The performance of CFO estimation with and without considering the effect of coupling is compared in Figure 6 . We can see that the spatial correlation introduced by the propagation environment degrades the MSE performance as compared to the i.i.d. channel. Mutual coupling adds additional degradation to the performance. This means that the detrimental effect due to the power loss (as shown in Figure 5 ) is larger than the beneficial effect due to reduced spatial correlation (as shown in Figure 4).

\section{CONCLUSION}

In this paper, we studied the effects of spatial correlation and antenna mutual coupling on the performance of CFO estimation in a MIMO system. We showed that Laplacian PAS introduces higher spatial correlation compared to a uniform PAS due to its narrower angular spread. For a Laplacian PAS with a constant angular spread, the spatial correlation is higher for larger mean AOA values. Computer simulations

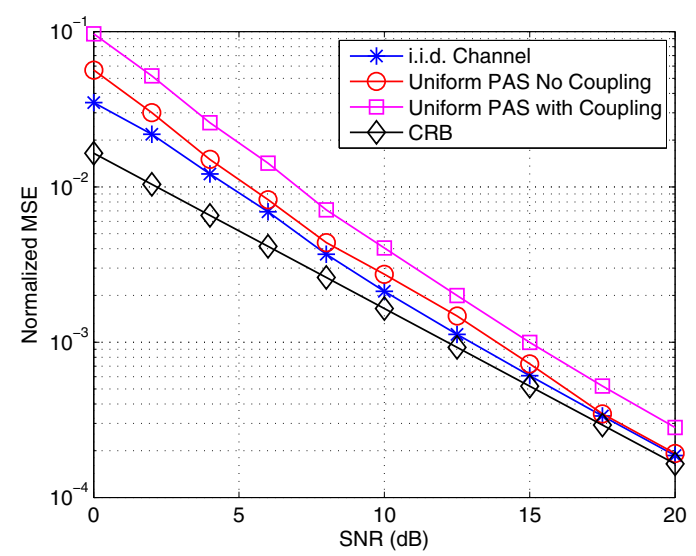

Fig. 6. Effects of mutual coupling on the performance of CFO estimation.

showed that the higher the spatial correlation, the higher the performance degradation in the CFO estimation compared to i.i.d. MIMO channels. We further showed that antenna mutual coupling has two effects. Firstly it reduces the spatial correlation, which is beneficial for CFO estimation. Secondly it introduces power loss on the received signal, which is detrimental. The overall effect of mutual coupling adds additional degradation to the performance of CFO estimation.

\section{REFERENCES}

[1] G. Foschini and M.J.Gans, "On limits of wireless communications in a fading environment when using multiple antennas," Wireless personal communications, vol. 6, no. 3, pp. 331-335, March 1998.

[2] I. Gupta and A. A. Ksienski, "Effect of mutual coupling on the performance of adaptive arrays," IEEE Trans. Antennas Propag., vol. AP-31, no. 5, pp. 785-791, Sept. 1983.

[3] R. Vaughan and J. Andersen, "Antenna diversity in mobile communications," IEEE Trans. Veh. Technol., vol. VT-36, no. 4, pp. 149-172, Nov. 1987.

[4] D. Shiu, G. Foschini, M. Gans, and J. Kahn, "Fading correlation and its effect on the capacity of multielement antenna sysetms," IEEE Trans. Commun., vol. 48, no. 3, pp. 502-513, Mar. 2000.

[5] B. Clerckx, C. Craeye, D. Vanhoenacker-Janvier, and C. Oestges, "Impact of antenna coupling on $2 \times 2$ MIMO communications," IEEE Trans. Veh. Technol., vol. 56, no. 3, pp. 1009-1018, May 2007.

[6] Q. Spencer, B. Jeffs, M. Jensen, and A. Swindlehurst, "Modeling the statistical time and angle of arrival characteristics of an indoor multipath channel," IEEE J. Sel. Areas Commun., vol. 18, no. 3, pp. 347-360, 2000.

[7] C. C. Chong, D. I. Laurenson, and S. McLaughlin, "Statistical characterization of the $5.2 \mathrm{GHz}$ wideband directional indoor propagation channels with clustering and correlation properties," in Proc. VTC 2002Fall Vehicular Technology Conference 2002 IEEE 56th, vol. 1, 24-28 Sept. 2002, pp. 629-633.

[8] V. Erceg and et.al., "TGn channel models," IEEE 802.11 document 802.11-03/940r4, May 2004

[9] L. Schumacher and B. Raghothaman, "Closed-form expressions for the correlation coefficient of directive antennas impinged by a multimodal truncated Laplacian PAS," IEEE Trans. Wireless Commun., vol. 4, no. 4, pp. 1351-1359, 2005.

[10] T. Schmidl and D. Cox, "Robust frequency and timing synchronization for OFDM," IEEE Trans. Commun., vol. 45, no. 12, pp. 1613-1621, Dec 1997.

[11] T.Svantesson, "The effects of mutual coupling using a linear array of thin dipoles of finite length," in IEEE SP Workshop on Statistical Signal and Array Processing, Sept. 1998, pp. 232-235.

[12] C. A. Balanis, Antenna theory : analysis and design, 2nd ed. John Wiley \& Sons, Inc., 1997. 